\title{
Molecular Docking Chlorogenic Acid and Isomer Compound as Cyclooxygenase-2 (COX-2) Inhibitor in Atherosclerosis
}

\author{
Ratna Nurarifa $^{1, *}$, Devi Era Rachmawati ${ }^{1}$, Dwi Utari ${ }^{1}$ \\ ${ }^{1}$ Department of Biology, Faculty of Mathematics and Natural Science, Brawijaya University, Veteran Street, Malang, East Java, \\ Indonesia, 65145
}

Submission: June 2020; Revised: July 2020; Accepted: October 2020

*Corresponding author: Ratna Nurafifa; e-mail: ratnan2297@ student.ub.ac.id ; tel.: +6285730895701

\begin{abstract}
Atherosclerosis was an inflammatory disease caused by oxidized LDL. Chloroghenic acid (CGA) was a polyphenol compound and its isomers such as Caffeoylquinic acid (CQA) and Feruloylquinic acid (FQA) can increase the activity of antioxidant activity and can significantly reduce total cholesterol in the blood. Cyclooxygenase (COX) was an enzyme that correlates with the inflammatory process. COX was divided into two isoforms, COX-1 and COX-2. Prostanoid synthesis via the action of cyclooxygenase-2 (COX-2) was a component of the inflammatory response. All prepared compound of Chlorogenic acid (CGA) and its isomer, Caffeoylquinic acid (CQA) and Feruloylquinic acid (FQA) was used for molecular docking by using HEX 8.0.0. Visualization process was done by using Discovery Studio Client 4.1 software. The results showed docking between COX-2 with CGA compounds and its isomers also drug Ibuprofen shows energy binding COX-2 - CGA of -320 kcal / mol, COX-2 - CQA of -298 kcal / mol, COX-2 - FQA of $282 \mathrm{kcal} / \mathrm{mol}$, and COX-2 - Ibuprofen of $-230 \mathrm{kcal} / \mathrm{mol}$. The docking results showed the CGA compound and its isomers have smaller energy bindings than the drug Ibuprofen. This showed that the CGA compound and its isomers were better to reduce COX-2 activity than the Ibuprofen drug so that it has the potential as an anti-inflammatory which can be used to prevent the formation of atherosclerosis.
\end{abstract}

Keywords: Atherosclerosis, Chlorogenic acid, COX-2, isomer, molecular docking

\section{INTRODUCTION}

Atherosclerosis is an inflammatory disease of blood vessels, especially arteries, characterized by the accumulation of fat in the intima tunica. This disease is closely related to high levels of Total Cholesterol (TC), Low-density Lipoprotein (LDL), triglycerides, and low levels of High-density Lipoprotein (HDL) in the body. The accumulation of large amounts of LDL molecules in the intima tunica causes the endothelial cells to stress and secrete adhesion compounds. Adhesion compounds will oxidize LDL, causing the formation of ox-LDL. This oxidized LDL triggers inflammation in blood vessels. Atherosclerosis can occur in arteries throughout the body [1].

Inflammation in the body is correlated with mediators such as Cyclooxygenase (COX). In this case COX plays a key role in inflammation as a rate-limiting enzyme in converting arachidonic acid to prostaglandins. COX is divided into two forms of isoforms derived from two separate genes, namely COX-1 and COX-2. COX-1 is constitutive of most tissues and mediates normal physiological functions. COX-2 is quickly induced at the inflammatory site. COX-2 is expressed by endothelial cells of smooth muscle cells and macrophages in atherosclerotic lesions. In vessel walls, monocytes differentiate into other macrophages that secrete cytokines and growth factors. Macrophage activation with interferon, lipopolysaccharide [2].

COX-2 inhibition has the potential to inhibit atherogenesis through anti-inflammatory effects. Aspirin and nonsteroidal anti-inflammatory drugs (NSAIDs) are the drugs most widely used to treat inflammatory diseases. The COX pathway is the main target of NSAIDs because COX catalyzes the fatty acid oxygenation to produce eicosanoids, which are the main sign of inflammation [3]. Ibuprofen (2- (4-isobutylphenyl) propanoic acid) has been introduced since around the 80 s is the least toxic NSAID and well tolerated by the body. The general anti-inflammatory properties of ibuprofen have been linked to COX inhibition. Ibuprofen provides pleiotropic effects such as inhibition of adhesion and migration of trans-endothelial leukocytes, suppressing the intracellular production of Reactive Oxygen Species (ROS), and modifying it ox-LDL [4].

Chlorogenic Acid (CGA) is a common phenolic compound and is processed into various foods and drinks. Previously phenolic compounds were reported to reduce COX activity and subsequently prevent the synthesis of eicosanoids. CGA compounds and their isomer compounds exhibit anti-inflammatory activity by reducing the activity of COX-2 by inhibiting prostaglandin synthesis [3]. Based on these theories, this study aims to clarify the relationship between CGA and its isomer compounds on atherosclerosis using molecular docking. Therefore, an in silico study was carried 
out through molecular docking that can predict the combination of CGA compounds and their isomers

\section{METHODS}

\section{Ligand and Protein Preparations}

$\begin{array}{ccc}\text { Chlorogenic } & \text { Acid } & \text { (CID1794427), } \\ \text { Feruloylquinic } & \text { Acid } & \text { (CID10133609), }\end{array}$

Caffeoylquinic Acid (CID10155706), and Ibuprofen control (CID3672) were obtained from the PubChem database with sdf file format. Then the receptor in the form of COX-2 protein (PDB ID: 5IKR) was obtained from Protein Data Bank. The ligand preparation involved minimizing bond energy and changing the sdf format to PDB format with the Open Babel tool in the PyRx Virtual Screening Tool software [5]. The 3-dimensional structure of the COX-2 receptor was prepared through Discovery Studio Visualizer [6]. Protein preparation was carried out to remove ligands and water molecules that attach to the protein's $3 \mathrm{D}$ structure.

\section{Molecular docking}

The docking process was carried out using Hex version 8.0.0 software by docking proteins' structure as receptors and compounds as ligands [7]. Chlorogenic acid and its isomers also Ibuprofen as control were docked with COX-2 so that there are four models of ligand and protein interactions. The results in the form of hex messages containing energy data were stored and the docking results visualized with Discovery Studio Client 4.1 Software.

\section{RESULTS AND DISCUSSIONS}

Based on the molecular docking results of Chlorogenic acid (CGA) and its isomers Caffeoylquinic acid (CQA) and Feruloylquinic acid (FQA) to COX-2 receptors, the energy binding of CGA is $-342.9 \mathrm{~kJ} / \mathrm{mol}$, energy binding of CQA is $298.5 \mathrm{~kJ} / \mathrm{mol}$. Energy binding of FQA is -282.9 $\mathrm{kJ} / \mathrm{mol}$ (Table 1). Based on the description above, it can be seen that the most effective compound to bind to COX-2 receptors was Chlorogenic acid which causes inhibition of COX-2 activity so that inflammation in atherosclerosis was inhibited. The results show that CGA and all isomers have lower bond energy than drug ibuprofen, a binding energy value of $-230.5 \mathrm{~kJ} / \mathrm{mol}$. The CGA and its analogues are potent inhibitors of $\mathrm{COX}-2$ receptor expression in inhibiting the formation of atherosclerosis. Binding affinity is the ability of a compound or drug to bind to a receptor [8]. Based on the number of hydrogen bonds, the strongest bond is found in the results of COX-2 docking with CGA because it by binding to $\mathrm{COX}-2$ receptors to see their potential for healing atherosclerosis.

has the most number of hydrogen bonds. It can be seen that the chlorogenic acid (CGA) compound has the greatest ability to inhibit COX-2 activity compared to other isomer compounds.

Tabel 1. Binding energy value of Chlorogenic acid isomers and Ibuprofen

\begin{tabular}{lll}
\hline No & Docking & Binding energy \\
\hline 1. & COX-2 - CGA & $-320 \mathrm{~kJ} / \mathrm{mol}$ \\
2. & COX-2 - CQA & $-298 \mathrm{~kJ} / \mathrm{mol}$ \\
3. & COX-2 - FQA & $-282 \mathrm{~kJ} / \mathrm{mol}$ \\
4. & COX-2 - IBP & $-230 \mathrm{~kJ} / \mathrm{mol}$ \\
\hline
\end{tabular}

Docking results of control Ibuprofen and all isomer compounds also show different interactions with COX-2. Ibuprofen has only one type of bond with the COX-2 receptor, the hydrophobic bond to the amino acids Pro153, Pro156, Pro153, and His39. The binding type is Alkyl except for His39 with Pi-Alkyl type. Docking results between Chlorogenic acid (CGA) compounds produce three types of bonds. There are eight hydrogen bonds, one hydrophobic bond, and one sulfur interaction. The Cys36 interacted with receptors with Pi-Alkyl type hydrophobic bonds. And there are other bonds with the Cys47 amino acid with Pi-Sulfur type and hydrogen bonding category with carbon-hydrogen bonding type. The next docking compound was the Caffeoylquinic Acid (CQA) compound, which has seven types of bonds with the COX-2 receptor. Conventional type hydrogen bonds occur in the amino acids His214, Lys215, and Phe210, whereas Asp213 carbon type hydrogen bonds. The His207 has been bound to COX-2 receptors in the Pi-Lone Pair category. Hydrophobic bonds only have one bond to the amino acid His207 with the Pi-Pi Stacked type. The last compound is Feruloylquinic Acid (FQA), with COX-2 receptors producing two types of bonds. There are three hydrogen bonds and two hydrophobic bonds. The bond that occurs with Gln46 has a conventional hydrogen bond. The Pro153 and Gly45 bonds are hydrogen bond types, and the bonds with Pro153 and Pro156 are hydrophobic bond types with the Pi-Alkyl type (Table 2). Docking visualization can be seen in Table 3.

The interaction of Ibuprofen with COX-2 is demonstrated by ligand binding with amino acid residues. The type of chemical bonds formed between ligands and amino acid residues is hydrophobic bonds. Amino acid residues are Pro153, Pro156, Pro153, and His39 and the bonds that occur are all hydrophobic bonds. The bond energy formed from Ibuprofen and COX-2 is -230 $\mathrm{kJ} / \mathrm{mol}$. 
Table 2. Interaction of Cyclooxygnase-2 (COX-2) and Ibuprofen, Chlorogenic acid, Caffeoylquinic acid, or Feruloylquinic acid

\begin{tabular}{|c|c|c|c|}
\hline Interaction & Name & Chemistry Bond & Types \\
\hline \multirow{4}{*}{ Ibuprofen } & :LIG1:C - B:PRO153 & Hydrophobic & Alkyl \\
\hline & :LIG1:C - B:PRO156 & Hydrophobic & Alkyl \\
\hline & :LIG1:C - B:PRO153 & Hydrophobic & Alkyl \\
\hline & B:HIS39 - :LIG1:C & Hydrophobic & Pi-Alkyl \\
\hline \multirow{10}{*}{$\begin{array}{l}\text { Chlorogenic acid - } \\
\text { COX-2 }\end{array}$} & A:ARG44:HE - :LIG1:O & Hydrogen Bond & Conventional Hydrogen Bond \\
\hline & A:GLN461:HE22 - :LIG1:O & Hydrogen Bond & Conventional Hydrogen Bond \\
\hline & A:ARG469:HE - :LIG1:O & Hydrogen Bond & Conventional Hydrogen Bond \\
\hline & A:GLY135:HA2 - :LIG1:O & Hydrogen Bond & Carbon Hydrogen Bond \\
\hline & A:PRO153:HD1 - :LIG1:O & Hydrogen Bond & Carbon Hydrogen Bond \\
\hline & A:ARG469:HD2 - :LIG1:O & Hydrogen Bond & Carbon Hydrogen Bond \\
\hline & :LIG1:H - A:CYS41:O & Hydrogen Bond & Carbon Hydrogen Bond \\
\hline & :LIG1:H - A:CYS41:O & Hydrogen Bond & Carbon Hydrogen Bond \\
\hline & A:CYS47:SG - :LIG1 & Other & Pi-Sulfur \\
\hline & :LIG1 - A:CYS36 & Hydrophobic & Pi-Alkyl \\
\hline \multirow{7}{*}{$\begin{array}{c}\text { Caffeoylquinic acid - } \\
\text { COX-2 }\end{array}$} & B:HIS214:HN - :LIG1:O & Hydrogen Bond & Conventional Hydrogen Bond \\
\hline & B:LYS215:HN - :LIG1:O & Hydrogen Bond & Conventional Hydrogen Bond \\
\hline & :LIG1:H - :LIG1:O & Hydrogen Bond & Conventional Hydrogen Bond \\
\hline & :LIG1:H - B:PHE210:O & Hydrogen Bond & Conventional Hydrogen Bond \\
\hline & B:ASP213:HA - :LIG1:O & Hydrogen Bond & Carbon Hydrogen Bond \\
\hline & :LIG1:O - B:HIS207 & Other & Pi-Lone Pair \\
\hline & B:HIS207 - :LIG1 & Hydrophobic & Pi-Pi Stacked \\
\hline \multirow{5}{*}{$\begin{array}{l}\text { Feruloylquinic acid - } \\
\text { COX-2 }\end{array}$} & A:GLN461:HE22 - :LIG1:O & Hydrogen Bond & Conventional Hydrogen Bond \\
\hline & A:PRO153:HD2 - :LIG1:O & Hydrogen Bond & Carbon Hydrogen Bond \\
\hline & :LIG1:H - A:GLY45:O & Hydrogen Bond & Carbon Hydrogen Bond \\
\hline & :LIG1 - A:PRO153 & Hydrophobic & Pi-Alkyl \\
\hline & :LIG1 - A:PRO156 & Hydrophobic & Pi-Alkyl \\
\hline
\end{tabular}

Note: Bold letter indicates the H-donor in hydrogen bond interaction, Alkyl in Alkyl, Sulfur in Pi-Sulfur, Pi-orbital in Pi-Alkyl and Pi-Pi stacked, and Lone Pair in Pi-Lone Pair.

The interaction of chlorogenic acid with COX-2 is shown by bonding with amino acid residues. Types of chemical bonds formed between ligands and amino acid residues are hydrogen bonds, hydrophobic bonds and other bonds. Interactions between ligands and proteins show 10 amino acid residues that correlate with chlorogenic acid. Amino acid residues are Arg44, Gln461, Arg469, Gly135, Pro153, Arg469, Cys41, Cys41, Cys36, and Cys47. The bonds that occur in amino acid residues Arg44, Gln46, Arg469, Gly135, Pro153, Arg469 are hydrogen bonds. The bond between the Cys36 amino acid residue and Lig1 is hydrophobic, and the bond between the Cys 27 amino acid residue and Lig 1 is another bond. The CGA - COX-2 complex proved binding energy is $-320 \mathrm{~kJ} / \mathrm{mol}$. The COX-2 docking with chlorogenic acid revealed a strong bond with high hydrogen bonds, so the bonds formed are also more stable.

Caffeoylquinoic acid-COX-2 showed hydrogen bonds, hydrophobic bonds, and other bonds. Interactions between ligands and proteins showed six amino acid residues, namely His 214 , Lys215, Phe210, Asp213, His207, His207. The bonds in residues of His214, Lys215, Phe210, Asp213 are hydrogen bonds, bonds in amino acid residues HIS207 are hydrophobic, and bonds in HIS207 residues are other bonds. The bond energy formed between CQA - COX-2 is $-298 \mathrm{~kJ} /$ mol. The COX-2 docking with caffeoylquinic acid has a lower number of hydrogen bonds than COX2 docking with CGA, so it can be seen that CQA also has a lower potential in inhibiting the activity of COX-2 formation compared to CGA.

The interaction of Feruloylquinic acid with COX-2 is shown by bonding with amino acids. 
The types of chemical bonds formed between ligands and amino acid residues are hydrogen bonds and hydrophobic bonds. Interactions between ligands and proteins show five amino acid residues that correlate with Feruloylquinic Acid. Amino acid residues consist of Gln461, Pro153, Gly45, Pro153, Pro156. The bonds that occur in the amino acid residues Gln461, Pro153, Gly45 are hydrogen bonds, whereas the amino acid residue bonds Pro153, Pro156 are hydrophobic. The bond energy formed between Feruloylquinic Acid and COX-2 is $-282 \mathrm{~kJ} / \mathrm{mol}$. Based on this, Feruloylquinic acid has the least amount of hydrogen bonds compared to CGA and CQA, so it can be seen that the bonds formed between COX-2 and Feruloylquinic acid have the lowest potential in inhibiting COX-2 expression in the formation of atherosclerosis.

Table 3. Docking Result with 4 ligands

\begin{tabular}{|c|c|c|c|}
\hline & Binding location & Ligand Interaction & 2D Diagram \\
\hline $\begin{array}{l}\text { COX-2 - } \\
\text { Ibuprofen }\end{array}$ & & & \\
\hline
\end{tabular}
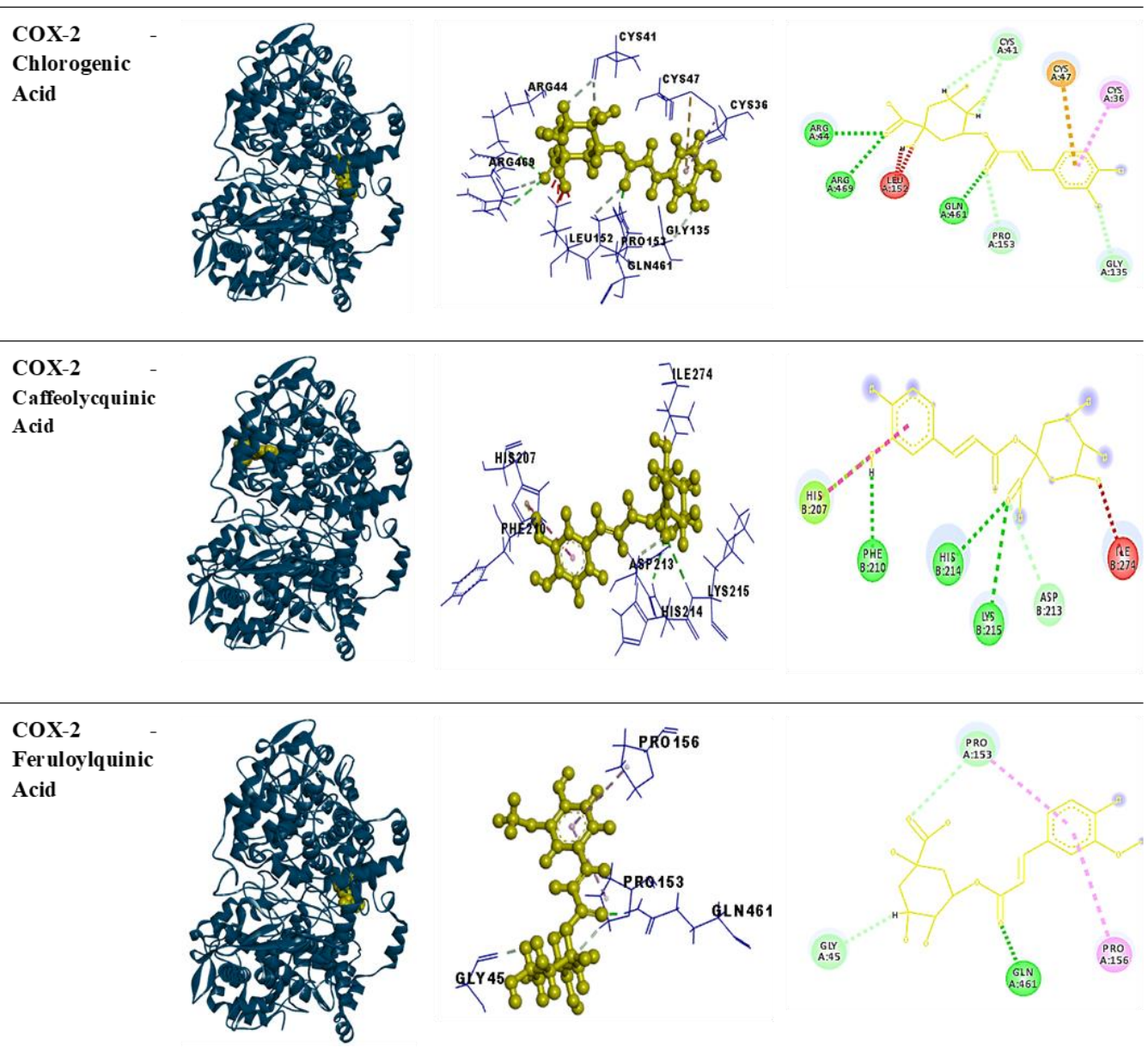

Note. Interaction between COX-2 and Ibuprofen, Chlorogenic acid, Caffeoylquinic acid, or Feruloylquinic acid is shown as the 3D binding location in the left column. The 3D ligand interactions between ligand and amino acids of COX-2 in the middle column. The right column shows $2 \mathrm{D}$ molecular interactions.

Based on the molecular docking results of CGA compounds and their isomers have more hydrogen bonds than Ibuprofen. Ibuprofen has only hydrophobic bonds in amino acid residue bonds, whereas CGA and its isomers have hydrogen and hydrophobic bonds, where hydrogen bonds dominate in amino acid residue bonds. Hydrogen bonds are more stable than 
hydrophobic bonds. The yield of bond energy produced by CGA and its isomers is lower compared to drug Ibuprofen. When the results of docking a receptor with a ligand show a lower binding energy value, it shows that the receptor with a ligand will be more easily bound. Docking is an interaction between the ligand and the receptor used to predict the ligand's position and orientation when bound to the receptor. From the docking process, the bond energy $(\Delta \mathrm{G})$ will be obtained, which is the stability parameter of the conformation between the ligand and the androgen receptor. Interacting ligands will tend to be in the lowest energy conditions. These conditions cause the molecule to be in a stable state, so the smaller the value of $\Delta \mathrm{G}$, the ligand's interaction with the receptor will be more stable. Molecular interactions on ligand-receptors include electrostatic interactions, hydrophobic interactions, and hydrogen bonds, contributing to the energy value of bonds $(\Delta \mathrm{G})$ of ligandreceptors [8].

The COX-2 and COX-1 have similar active sites with high structure similarity. There are only two different amino acids within the isoforms. Recent studies discovered active sites within the residues, Arg210, Leu352, Tyr355, Tyr348, Trp387, Ser530, and Leu531 [9]. Recently study shown that Ser-530 was homolog in COX-1 and COX-2 indicated that NSAID was non-selected inhibitors for COX-2 [10]. Interestingly, our study found another active site that binds Chlorogenic acid, Feruloylquinic acid, and Caffeoylquinic acid. Chlorogenic acid interacted with COX-2 in binding sites Arg44, Gln461, Arg469, Gly135, Pro153, and Cys41. Feruloylquinic acid inactivated COX-2 by the interactions in Gln461, Pro153, and Gly45. Caffeoylquinic acid inhibits COX-2 in His214, Lys215, Asp213 and Phe210.

COX-2 is involved in the synthesis of Arachidonic acid into Prostaglandin (PG), leading to atherosclerosis. After docking chlorogenic acid and its isomers, the smallest energy binding is shown by chlorogenic acid compound. Chlorogenic acid successfully binds to COX-2 has the effect of inhibiting Arachidonic acid synthesis by COX-2. Successful COX-2 inhibition can also reduce prostaglandin production (PGI) as an athero-protective effect that balances the synthesis of prostaglandins by COX-2 [9]. Therefore, Chlorogenic acid is predicted as a potential COX2 enzyme inhibitor.

Inflammation that occurs in the body is correlated with inflammatory mediators such as COX-2. COX-2 has the function of initiating and maintaining physiological conditions during inflammation [11]. Compare to affinity character between chlorogenic acid and COX-2 in this research indicated that interaction was blocking at
COX2 in different active site location compare to other ligands. Therefore Chlorogenic acid and isomers are competitive inhibitors [12]. Different binding sides in COX-2 have a potential role for inhibiting COX-2 function in different effects compared to chemical drugs. CGA and its isomers have an affinity with the different COX-2 active sites in amino acid residues that bind to hydrogen bonds, causing blocking of inflammatory pathways such as NF-K $\beta$. Chlorogenic acid significantly inhibited the activity of COX-2 without any cytotoxicity. Chlorogenic acid also attenuated pro-inflammatory cytokines (including IL-1 $\beta$ and TNF- $\alpha$ ) and other inflammation-related markers such as IL-6 in a dose-dependent manner. Finally, chlorogenic acid inhibited the nuclear translocation of NF- $\kappa \mathrm{B}$. Chlorogenic acid may be beneficial for the prevention and treatment of antiinflammatory diseases [13]. So that when CGA and isomers bind to COX-2, inflammation can be reduced and the formation of atherosclerosis can be stopped.

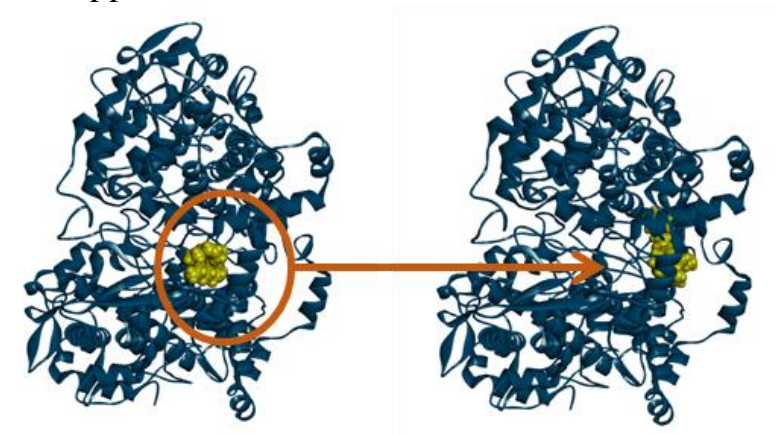

Figure 1. Arachidonic acid binds into another site of COX-2

\section{CONCLUSION}

Based on research by docking of COX-2 and chlorogenic acid (CGA) compounds and its isomers namely caffeoylquinic acid (CQA) and Feruloylquinic acid (FQA) and Ibuprofen drugs, it is known that the strongest bond is found in the docking results of COX-2 with chlorogenic acid (CGA). The smallest binding energy value was also obtained from the results of COX-2 docking with Chlorogenic acid (CGA) compared to its isomer compound and Ibuprofen drug. This shows that Chlorogenic acid (CGA) is predicted as a potential inhibitor of COX-2 activity in inhibiting atherosclerosis formation.

\section{ACKNOWLEDGEMENT}

The authors are sending our regards to Prof. Dra Fatchiyah, M.Kes., Ph.D., Prof. Widodo, Ph.D.Med.Sc., Nia Kurniawan, S.Si, M.P., D.Sc., and Yoga Dwi Jatmiko, S.Si., M.App.Sc., Ph.D. and our thanks to all laboratory assistant for the guidance on this research. 


\section{REFERENCES}

1. Galkina E, Ley K. Immune and inflammatory mechanisms of atherosclerosis. Annu Rev Immunol. 2009; 27:165-97. doi: 10.1146/annurev.immunol.021908.132620.

2. Burleigh, M.E., Vladimir, R.B., John, A.O., Raymond C.H., Shiva, G., Denis, R., Lawrence, J.M., Jason, D.M., Sergio, F., and MacRae, F.L. 2002. Cyclooxygenase-2 Promotes Early Atherosclerosis Lesion Formation in LDL Receptor-Deficient Mice. Circulation, $\quad 105(15)$ : 1816-1823. doi:10.1161/01.CIR.0000014927.74465.7F.

3. Liang N, Kitts DD. Role of Chlorogenic Acids in Controlling Oxidative and Inflammatory Stress Conditions. Nutrients. 2015 Dec 25;8(1):16. doi: 10.3390/nu8010016.

4. Zapolska-Downar D, Naruszewicz M. A pleiotropic antiatherogenic action of ibuprofen. Med Sci Monit. 2001;7(4):837-41.

5. Dallakyan, N. and A.J. Olson. Methods in molecular Biology. 2015. 1263, 243-250.

6. Dassault system BIOVIA, BIOVIA Discovery studio 2019. San Diego 2019: Dassaults System.

7. Ritchie, D.W. Bioinformatics. 2008. 24(17):1865-73.

8. Muttaqin, F. Z., Halim Ismail, Hubbi N. M. 2019. Studi Molecular Docking, Molecular
Dynamic, dan Prediksi Toksisitas Senyawa Turunan Alkaloid Naftiridin Sebagai Inhibitor Protein Kasein Kinase 2- $\alpha$ Pada Kanker Leukemia. Pharmacoscript 2(1):49-64. doi:10.36423/pharmacoscript.v2i1.241.

9. Chen, Y., and Kuntze, C. 2007. Novel selective inhibitors of hydroxyxanthone derivatives for human cyclooxygenase-2. Acta Pharmacology. 28 (12): 2027-2032.

10.Hamzah, N., Aahmad, N., Nurshalati, T., Ika, M. 2015. Studi Farmakor Reseptor COX-2 sebagai Anti-inflamasi. Jurnal Farmasi, 2(3):99-107.

11.Bare, Y., Dewi, R.T.S., Yoga, T.R., Gabriella, C.K., Agustina, E., and Andri M. 2016. InSilico Insight the Prediction of Chlorogenis Acid in Coffe Trough Cyclooxigenase-2 (COX-2) Interaction. Biogenesis 7(2): 100105.

12.Perez YR, Alvarez D, Combariza A. 2019. Ligand-Protein Interactions: A Hybrid ab initio/Molecular Mechanics Computational Study. Preprints. 1: 1-24.

13.Hwang SJ, Kim YW, Park Y, Lee HJ, Kim KW. Anti-inflammatory effects of chlorogenic acid in lipopolysaccharide-stimulated RAW 264.7 cells. Inflamm Res. 2014 Jan;63(1):8190. doi: 10.1007/s00011-013-0674-4. 